\title{
TWO-PARAMETER GROUPS OF FORMAL POWER SERIES
}

\author{
BY \\ ABSELOM RAN $\left.{ }^{1}\right)$
}

Summary. By $\Omega^{F}$ we denote the group of the formal power series having the form $F(z)=\sum_{q=1}^{\infty} f_{q} z^{q}, f_{1} \neq 0$, with respect to formal composition of power series.

The problem of analytic iteration leads to the study of subgroups of $\Omega^{F}$, having the form

$$
F(z, s)=\sum_{q=1}^{\infty} f_{q}(s) z^{q}
$$

where the coefficients $f_{q}(s)$ are analytic functions of the complex parameter $s$, such that for any two complex numbers $s$ and $t$ the formal law of composition

$$
F[F(z, s), t]=F(z, s+t)
$$

is valid [6], [8].

The purpose of the present paper is to study similar two-parameter subgroups of $\Omega^{F}$. In $\S 1 r$-parameter analytic subgroups of $\Omega^{F}$ are defined, as well as other concepts connected with the problem. In $\$ 2$ the importance of two-parameter subgroups is emphasized. It is shown that the number of parameters of analytic subgroups of $\Omega^{F}$ can always be reduced to two at most. The existence of a countable number of classes of the two-parameter subgroups of $\Omega^{F}$ is shown. $\$ 3$ gives the explicit form of the coefficients $f_{q}\left(a^{1}, a^{2}\right)$ of a two-parameter subgroup of $\Omega^{F}$ :

$$
F\left(z, a^{1}, a^{2}\right)=\sum_{q=1}^{\infty} f_{q}\left(a^{1}, a^{2}\right) z^{q}
$$

In $\$ 4$ the existence of canonical representations for two-parameter analytic subgroups of $\Omega^{F}$ is proven, and it is shown that every two-parameter analytic subgroup of $\Omega^{F}$ is globally isomorphic to one of the groups

$$
H_{n}\left(z, a^{1}, a^{2}\right)=\left(1+a^{1}\right) z /\left(1+a^{2} z^{n}\right)^{1 / n}, \quad n=1,2, \ldots
$$

(no two of which are globally isomorphic to each other).

Received by the editors March 20, 1969.

( $\left.{ }^{1}\right)$ This paper is partially based on a part of the author's thesis for the D.Sc. degree, submitted to the Senate of the Technion, Israel Institute of Technology. The author wishes to express his deepest gratitude to Professor E. Jabotinsky for his helpful guidance.

Copyright d 1969, American Mathematical Society 
1. Introduction and definitions.

1.1. Let $\Sigma^{F}$ denote the linear algebra of formal power series (over the field of complex numbers) having the form

$$
F(z)=\sum_{q=0}^{\infty} f_{q} z^{q}
$$

(Operations on formal power series are defined in [2, Chapter 1].) $\Sigma^{F}$ is equipped with the metric

for $F, G \in \Sigma^{F}$.

$$
\rho(F, G)=\sum_{q=0}^{\infty} 2^{-q} \frac{\left|f_{q}-g_{q}\right|}{1+\left|f_{q}-g_{q}\right|}
$$

Let

$$
F(z, s)=\sum_{q=0}^{\infty} f_{q}(s) z^{q}
$$

be a family of formal power series depending on a complex parameter $s, s \in D$, $D$ being a domain in the complex plane. We say, that $F(z, s)$ is analytically dependent on $s$, if the limit

$$
\frac{\partial F(z, s)}{\partial s}=\lim _{h \rightarrow 0} \frac{F(z, s+h)-F(z, s)}{h}=\sum_{q=0}^{\infty} f_{q}^{\prime}(s) z^{q}
$$

exists in the metric of $\Sigma^{F}$ for every $s \in D$. This limit exists if and only if all the coefficients $f_{q}(s)$ are analytic functions of $s$ in $D$.

Let $C^{r}$ denote the $r$-dimensional complex space. $\mathbf{a} \in C^{r}$ denotes a vector with $r$ complex components: $\mathbf{a}=\left(a^{1}, a^{2}, \ldots, a^{r}\right)$. A family of formal power series

$$
F(z, \mathbf{a})=\sum_{q=0}^{\infty} f_{q}(\mathbf{a}) z^{q}
$$

is said to be analytically dependent on a in a domain $D$ of $C^{r}$, if $F(z$, a) is a continuous function from $D$ into $\Sigma^{F}$, and is analytically dependent on each of the components of a. $F(z, \mathbf{a})$ is thus analytically dependent on $\mathbf{a}$, if and only if all the coefficients $f_{q}(\mathbf{a}), q=0,1,2, \ldots$ are analytic functions of the components of $\mathbf{a}$ in $D$.

1.2. Let $\Omega^{F}$ denote the subset of $\Sigma^{F}$, which contains the power series of the form

$$
F(z)=\sum_{q=1}^{\infty} f_{q} z^{q}, \quad f_{1} \neq 0 .
$$

$\Omega^{F}$ forms a topological group with respect to formal substitution of formal power series, and the topology induced in $\Omega^{F}$ from $\Sigma^{F}$.

The group $\Omega^{F}$ can be represented by infinite matrices. Indeed, for every $F(z) \in \Omega^{F}$ and every integer $m>0$ put

$$
[F(z)]^{m}=\sum_{q=1}^{\infty} f_{m, q} z^{q}
$$


The matrix $\left\|f_{m, q}\right\|, 1 \leqq m, q<\infty$ represents the series $F(z)$ in the sense, that if the matrices $\left\|f_{m, q}\right\|$ and $\left\|g_{m, q}\right\|$ represent the series $F(z)$ and $G(z)$ respectively, then the series $F[G(z)]$ is represented by the matrix $\left\|f_{m, q}\right\| \times\left\|g_{m, q}\right\|$, where $\times$ represents matrix multiplication ([5], [9]).

By $\Omega$ we denote the subgroup of $\Omega^{F}$ constituted by the power series of $\Omega^{F}$ with a nonzero radius of convergence.

1.3. A subgroup of $\Omega^{F}$ is said to be an analytic r-parameter subgroup of $\Omega^{F}$, if its elements can be written as an $r$-parameter family of $\Omega^{F}: F(z, \mathbf{a})=F\left(z, a^{1}, \ldots, a^{r}\right)$, where a ranges in some domain $D$ of $C^{r}$, so that:

(1) $F(z, a)$ is analytically dependent on $\mathbf{a}$.

(2) There is a vector function $\phi(\mathbf{a}, \mathbf{b})$, that is, $r$ scalar functions of $2 r$ variables

$$
\phi^{1}\left(a^{1}, \ldots, a^{r}, b^{1}, \ldots, b^{r}\right), \ldots, \phi^{r}\left(a^{1}, \ldots, a^{r}, b^{1}, \ldots, b^{r}\right)
$$

analytic in $a^{1}, \ldots, a^{r}, b^{1}, \ldots, b^{r}$ for $\mathbf{a}, \mathbf{b} \in D$, which is called the "multiplication table", such that

$$
F[F(z, \mathbf{a}), \mathbf{b}]=F[z, \phi(\mathbf{a}, \mathbf{b})]
$$

holds for every $\mathbf{a}, \mathbf{b} \in D$.

An analytic $r$-parameter subgroup of $\Omega^{F}$ is thus a connected complex Lie group. The parametrization of such a group is not unique. We shall, for convenience, consider only parametrizations satisfying: $F(z, 0)=z$, which implies $0 \in D$. It follows, that $\phi(\mathbf{a}, \mathbf{b})$ should satisfy

$$
\phi(\mathbf{0}, \mathbf{b})=\mathbf{b}, \quad \boldsymbol{\phi}(\mathbf{a}, \mathbf{0})=\mathbf{a} .
$$

By a local r-parameter analytic subgroup of $\Omega^{F}$ we call a family of elements of $\Omega^{F}$, $F(z, a)$, analytically dependent on a in some fixed connected neighborhood $D$ of $\mathbf{a}=\mathbf{0}$ in $C^{r}$, with $F(z, 0)=z$, such that (5) is satisfied with respect to some multiplication table $\phi(\mathbf{a}, \mathbf{b})$, analytic in $a^{1}, \ldots, a^{r}, b^{1}, \ldots, b^{r}$, whenever $\mathbf{a}, \mathbf{b}$, and $\boldsymbol{\phi}(\mathbf{a}, \mathbf{b})$ belong to $D$.

A local $r$-parameter analytic subgroup of $\Omega^{F}$ is thus a complex connected local Lie group. We note, that every connected neighborhood of the identity element of an analytic $r$-parameter subgroup of $\Omega^{F}$ forms a local subgroup.

1.4. Let $F(z, a)$ be an analytic $r$-parameter local or global subgroup of $\Omega^{F}$, with the multiplication table $\phi(\mathbf{a}, \mathbf{b})$. We define

$$
V_{j}^{i}(\mathbf{a})=\left(\partial \phi^{i}(\mathbf{a}, \mathbf{b}) / \partial b^{j}\right)_{\mathbf{b}=0} .
$$

Because of (6) we have

$$
V_{j}^{i}(\mathbf{0})=\delta_{j}^{i} .
$$

The structure constants of the subgroup are given by

$$
c_{j k}^{i}=\left(\frac{\partial v_{j}^{i}(\mathbf{a})}{\partial a^{k}}\right)_{\mathbf{a}=0}-\left(\frac{\partial v_{k}^{i}(\mathbf{a})}{\partial a^{j}}\right)_{\mathbf{a}=0}
$$


we note that for a commutative group $\phi(\mathbf{a}, \mathbf{b})=\phi(\mathbf{b}, \mathbf{a})$ and from (7) and (9) follows $c_{j k}^{i}=0,1 \leqq i, j, k \leqq r$.

1.5. Let $F(z, a)$ be an analytic $r$-parameter local or global subgroup of $\Omega^{F}$. Denote

$$
L_{j}(z)=\left(\frac{\partial F(z, \mathbf{a})}{\partial a^{j}}\right)_{\mathbf{a}=0}=\sum_{q=0}^{\infty} l_{q}^{(j)} z^{q+1}, \quad j=1, \ldots, r .
$$

(Note that the $L_{j}(z)$ have no free terms.)

We define the tangent space to the subgroup $F(z, \mathbf{a})$ to be the linear subspace of $\Sigma^{F}$ generated by $\left\{L_{j}(z)\right\}_{j=1}^{r}$. We note that the tangent space is invariant under regular analytic transformations of the group parameter a. Indeed, let another $r$-dimensional parameter $\mathbf{s}$ be defined by the analytic function $\mathbf{a}=\mathbf{a}(\mathbf{s})$ with $\left(\partial\left(a^{1}, \ldots, a^{r}\right) / \partial\left(s^{1}, \ldots, s^{r}\right)\right)_{\mathbf{s}=0} \neq 0$ and $\mathbf{0}=\mathbf{a}(\mathbf{0})$. Put

$$
F^{*}(z, \mathbf{s})=F[z, \mathbf{a}(\mathbf{s})] .
$$

Differentiating the last equation we get

$$
L_{j}^{*}(z)=\left(\frac{\partial F^{*}(z, \mathbf{s})}{\partial s^{j}}\right)_{\mathbf{s}=0}=\sum_{q=1}^{r}\left(\frac{\partial a^{q}}{\partial s^{j}}\right)_{\mathbf{s}=0} \cdot\left(\frac{\partial F(z, \mathbf{a})}{\partial a^{q}}\right)_{\mathbf{a}=0}=\sum_{q=1}^{r}\left(\frac{\partial a^{q}}{\partial s^{j}}\right)_{\mathbf{s}=0} \cdot L_{q}(z)
$$

and hence the $\left\{L_{j}^{*}(z)\right\}$ generate the same subspace of $\Sigma^{F}$ as the $\left\{L_{q}(z)\right\}$.

1.6. We shall now establish a system of formal differential equations in $\Sigma^{F}$ for a given $r$-parameter analytic subgroup of $\Omega^{F}, F(z, \mathbf{a})$, having the multiplication table $\phi(\mathbf{a}, \mathbf{b})$ and find the integrability conditions of this system.

Differentiating (5) with respect to $b^{j}$ and putting $\mathbf{b}=\mathbf{0}$ we obtain

$$
L,[F(z, \mathbf{a})]=\sum_{k=1}^{r} \frac{\partial F(z, \mathbf{a})}{\partial a^{k}} V_{j}^{k}(\mathbf{a}), \quad 1 \leqq j \leqq r .
$$

In order to obtain the integrability conditions of this system we differentiate (11) with respect to $a^{i}$ and put $\mathbf{a}=\mathbf{0}$. We get, using (8)

$$
L_{j}^{\prime}(z) L_{i}(z)=\left(\frac{\partial^{2} F(z, \mathbf{a})}{\partial a^{i} \partial a^{j}}\right)_{\mathbf{a}=0}+\sum_{k=1}^{r} L_{k}(z)\left(\frac{\partial v_{j}^{k}(\mathbf{a})}{\partial a^{i}}\right)_{\mathbf{a}=0} .
$$

Interchanging the indices $i$ and $j$, we get

$$
L_{i}^{\prime}(z) L_{j}(z)=\left(\frac{\partial^{2} F(z, \mathbf{a})}{\partial a^{j} \partial a^{i}}\right)_{\mathbf{a}=0}+\sum_{k=1}^{r} L_{k}(z)\left(\frac{\partial v_{i}^{k}(\mathbf{a})}{\partial a^{j}}\right)_{\mathbf{a}=0} .
$$

Subtracting (13) from (12), using (9), we get

$$
L_{j}^{\prime}(z) L_{i}(z)-L_{j}(z) L_{i}^{\prime}(z)=\sum_{k=1}^{r} c_{j i}^{k} L_{k}(z), \quad 1 \leqq i, j \leqq r .
$$

We shall refer to the system (14) as the integrability conditions of the group $F(z, \mathbf{a})$. 
2. Essential parameters and classification of analytic subgroups of $\Omega^{F}$.

2.1. Let $F(z, \mathbf{a})=F\left(z, a^{1}, \ldots, a^{r}\right)$ be an analytic $r$-parameter local or global subgroup of $\Omega^{F}$. The parameters $a^{1}, \ldots, a^{r}$ are said to be essential, if the group $F(z$, a) cannot be represented as an $(r-1)$-parameter group; that is, if it impossible to find $r-1$ analytic functions: $A^{j}=A^{j}\left(a^{1}, \ldots, a^{r}\right), 1 \leqq j \leqq r-1$ such that

$$
F\left(z, a^{1}, \ldots, a^{r}\right) \equiv F\left(z, A^{1}, \ldots, A^{r-1}\right) .
$$

We cite the following criterion for the parameters $a^{1}, \ldots, a^{r}$ to be essential [3, Chapter 1, §3]:

Let $F(z, \mathbf{a})=F\left(z, a^{1}, \ldots, a^{r}\right)$ be a family of series of $\Omega^{F}$, analytically dependent on $a^{1}, \ldots, a^{r}$. A necessary and sufficient condition for the parameters $a^{1}, \ldots, a^{r}$ to be essential is that the equation

$$
\sum_{k=1}^{r} \psi_{k}(\mathbf{a}) \frac{\partial F(z, \mathbf{a})}{\partial a^{k}} \equiv 0
$$

where $\psi_{k}(\mathbf{a})$ are analytic functions of $a^{j}, 1 \leqq j \leqq r$, be satisfied identically for all a if and only if $\psi_{k}(\mathbf{a}) \equiv 0$ for $k=1, \ldots, r$ for all $\mathbf{a}$.

As a direct consequence we have:

LEMMA 1. Let $F\left(z\right.$, a) be an analytic r-parameter local or global subgroup of $\Omega^{F}$. If for some $j$,

$$
L_{j}(z)=\left(\frac{\partial F(z ; \mathbf{a})}{\partial a^{j}}\right)_{\mathbf{a}=0} \equiv 0
$$

then the group parameters $a^{1}, \ldots, a^{r}$ are not essential.

Proof. From (16) and (11) it follows that:

$$
\sum_{k=1}^{r} V_{j}^{k}(\mathbf{a}) \frac{\partial F(z, \mathbf{a})}{\partial a^{k}} \equiv 0
$$

while from (8) we know that $V_{j}^{j}(0)=1 \neq 0$; hence from the cited criterion follows that the parameters $a^{1}, \ldots, a^{r}$ are not essential.

2.2. We shall show now, that the maximal number of essential parameters in an analytic local or global subgroup of $\Omega^{F}$ is 2 . This results from a theorem due to L. Bianchi [1]; we bring, though, an elementary proof due to E. Jabotinsky.

THEOREM 1. Let $F\left(z, a^{1}, \ldots, a^{r}\right)$ be an analytic $r$-parameter local or global subgroup of $\Omega^{F}$. If the parameters $a^{1}, \ldots, a^{r}$ are essential, then $r \leqq 2$.

Proof. Let $F\left(z, a^{1}, \ldots, a^{r}\right)$ be an analytic $r$-parameter local or global subgroup of $\Omega^{F}$, where $a^{1}, \ldots, a^{r}$ are essential parameters. By a regular linear transformation of the parameters:

$$
a^{j}=\sum_{i=1}^{r} \alpha_{i j} a^{* i}, \quad j=1, \ldots, r
$$


the group will have a new parametrization:

$$
F^{*}\left(z, a^{* 1}, \ldots, a^{* r}\right) \equiv F\left(z, a^{1}, \ldots, a^{r}\right) .
$$

The tangent vectors $L_{j}^{*}(z)$ are linear combinations of the $L_{k}(z)$ :

$$
L_{j}^{*}(z)=\left(\frac{\partial F^{*}\left(z, \mathbf{a}^{*}\right)}{\partial a^{* j}}\right)_{\mathbf{a}^{*}=0}=\sum_{k=1}^{r}\left(\frac{\partial a^{k}}{\partial a^{* j}}\right)_{\mathbf{a}^{*}=0}\left(\frac{\partial F(z, \mathbf{a})}{\partial a^{k}}\right)_{\mathbf{a}=0}=\sum_{k=1}^{r} \alpha_{k j} L_{k}(z) .
$$

By a suitable choice of the matrix $\left\|\alpha_{i, j}\right\|$ we can obtain a system $\left\{L_{j}^{*}(z)\right\}$ in which the lowest power of $z$ with a nonzero coefficient will be different in any two of the series $L_{j}^{*}(z)$, and that in every one of the series $L_{j}^{*}(z)$ the first nonzero coefficient will be equal to 1 , that is, for every $j$ we will have: $L_{j}^{*}(z)=z^{p_{j}}+\cdots$, where $i \neq j$ implies $p_{i} \neq p_{j}$. The assumption that the parameters are essential implies, by Lemma 1 , that $L_{j}^{*}(z) \not \equiv 0$ for $1 \leqq j \leqq r$. Let $i$ be the index for which $p_{i}=\max \left\{p_{1}, \ldots, p_{r}\right\}$. The integrability condition of the group (14) for this $i$ and any $j \neq i$ is:

$$
L_{i}^{*}(z) L_{j}^{* \prime}(z)-L_{i}^{* \prime}(z) L_{j}^{*}(z)=\sum_{k=1}^{r} c_{j i}^{* k} L_{k}^{*}(z)
$$

Equate now the lowest powers of $z$ in both sides of (17). On the left-hand side the lowest power of $z$ with a nonzero coefficient is:

$$
p_{j} z^{p_{i}} z^{p_{j}-1}-p_{i} z^{p_{j} z^{p_{i}-1}}=\left(p_{j}-p_{i}\right) z^{p_{i}+p_{j}-1} .
$$

If we denote by $z^{p_{q}}$ the lowest power of $z$ with a nonzero coefficient on the right-hand side of (17) we get:

$$
p_{i}+p_{j}-1=p_{q}
$$

From $p_{i}=\max \left\{p_{1}, \ldots, p_{r}\right\}$ it follows that $p_{j}-1 \leqq 0$ or, as $L_{j}^{*}(z)$ has no constant term: $p_{j}=1$. This should hold for every $j, j \neq i$; so all the $L_{j}^{*}(z), j \neq i$, should start with the term $z$. But as each of the $L_{j}^{*}(z)$ starts with a different power of $z$, there may exist only one $L_{j}^{*}(z)$ in addition to $L_{i}^{*}(z)$, and hence $r \leqq 2$, completing the proof.

2.3. The one-parameter analytic subgroups of $\Omega^{F}$ were studied by E. Jabotinsky [6] and by the present author [8]. It turns out that although all the one-parameter analytic subgroups are locally isomorphic, there are two types of such groups, which are not globally isomorphic: The simply connected type and the nonsimply connected type.

In view of Theorem 1 there remains only the study of two-parameter analytic subgroups of $\Omega^{F}$. There are only two types of two-dimensional Lie groups which are not locally isomorphic: The commutative groups and the noncommutative groups [1]. In a commutative two-parameter analytic subgroup of $\Omega^{F}$ the parameters are not essential. Indeed, as all the structure constants (9) of a commutative group are zero, the integrability condition (14) of the group takes the form:

$$
L_{1}^{\prime}(z) L_{2}(z)-L_{2}^{\prime}(z) L_{1}(z)=0
$$


and this implies $L_{1}(z)=k L_{2}(z)$, where $k$ is a complex number. After a linear transformation of the parameters:

$$
a^{1}=a^{* 1}, \quad a^{2}=-k a^{* 1}+a^{* 2}
$$

we get $L_{1}^{*}(z)=0$, and hence, by Lemma 1 , the group parameters are not essential.

2.4. There thus remains to be studied only the case of the noncommutative two-parameter subgroups. All noncommutative two-dimensional Lie groups are locally isomorphic, and one can, by transformation of the parameters in some neighborhood of the identity element of a noncommutative two-parameter analytic local subgroup of $\Omega^{F}$, bring its multiplication table to the form:

$$
\phi_{n}^{1}\left(a^{1}, a^{2}, b^{1}, b^{2}\right)=a^{1}+b^{1}+a^{1} b^{1}, \quad \phi_{n}^{2}\left(a^{1}, a^{2}, b^{1}, b^{2}\right)=a^{2}+b^{2}\left(1+a^{1}\right)^{n},
$$

where $n$ is a fixed positive integer. The multiplication table $\phi_{n}(\mathbf{a}, \mathbf{b})$ defined by (19) shall be called the standard multiplication table of type $n$. An example of a global two-parameter analytic subgroup of $\Omega^{F}$ having the multiplication table (19) is given by the series representing:

$$
F\left(z, a^{1}, a^{2}\right)=\left(1+a^{1}\right) z /\left(1+a^{2} z^{n}\right)^{1 / n}
$$

where the parameters range in the domain: $D=\left\{\left(a^{1}, a^{2}\right) \mid a^{1} \neq-1\right\}$. (This example shows that $\phi_{n}(\mathbf{a}, \mathbf{b})$ defined by (19) really define a multiplication table of a group.) An example of a local two-parameter analytic subgroup of $\Omega^{F}$ having the standard multiplication table of type 1 is given by

$$
G\left(z, a^{1}, a^{2}\right)=\left(1+a^{1}\right)^{1 / 2} z /\left(1+a^{2} z^{2}\right)^{1 / 2}
$$

where the parameters range in the domain $B=\left\{\left(a^{1}, a^{2}\right)|| 1+a^{1} \mid<1\right\}$.

2.5. Let $F\left(z, a^{1}, a^{2}\right)$ be a two-parameter analytic subgroup of $\Omega^{F}$, having the multiplication table $\phi_{n}(\mathbf{a}, \mathbf{b})$ given by (19). From (7) we get

$$
V_{1}^{1}(\mathbf{a})=1+a^{1}, \quad V_{2}^{1}(\mathbf{a})=0, \quad V_{1}^{2}(\mathbf{a})=0, \quad V_{2}^{2}(\mathbf{a})=\left(1+a^{1}\right)^{n} .
$$

From here and from (9) we get the structure constants

$$
c_{1,1}^{1}=c_{2,2}^{1}=c_{1,1}^{2}=c_{2,2}^{2}=c_{1,2}^{1}=c_{2,1}^{1}=0, \quad c_{1,2}^{2}=-n, \quad c_{2,1}^{2}=n .
$$

The integrability condition (14) becomes:

$$
L_{2}^{\prime}(z) L_{1}(z)-L_{1}^{\prime}(z) L_{2}(z)=n L_{2}(z) .
$$

We shall use equation (20) to obtain some information about the $L_{i}(z), i=1,2$. Using the notation (10) for the terms of $L_{i}(z)$, we shall compare the lowest powers of $z$ with nonzero coefficients on both sides of (20). Suppose that the first nonzero coefficient of $L_{1}(z)$ is $l_{r-1}^{(1)}$ (the coefficient of $z^{r}$ ) and the first nonzero coefficient of $L_{2}(z)$ is $l_{s-1}^{(2)}$. The first nonzero term on the right in (20) is tiien $n l_{s-1}^{(2)} z^{s}$. The first nonzero term on the left in $(20)$ is

$$
(s-r) l_{s-1}^{(2)} l_{r-1}^{(1)} z^{s+r-1} .
$$


We note that the coefficient of this term cannot vanish, because otherwise we get by comparing with the other side of $(20): s+r-1<s$, that is, $r<1$, which is impossible, as the $L_{i}(z)$ have no constant terms. Hence

$$
(s-r) l_{s-1}^{(2)} l_{r-1}^{(1)} z^{s+r-1}=n l_{s-1}^{(2)} z^{s} .
$$

Comparing the powers of $z$ we get $r=1$, that is, $l_{0}^{(1)} \neq 0$. Putting $r=1$ into (21) and dividing by $l_{s-1}^{(2)}$ (which, by assumption is not 0 ) we get

$$
(s-1) l_{0}^{(1)}=n
$$

hence $s>1$, that is, $l_{0}^{(2)}=0$.

We say, that a two-parameter analytic (local or global) subgroup of $\Omega^{F}$, having the multiplication table (19) is of class $m$, if $l_{0}^{(2)}=l_{1}^{(2)}=\cdots=l_{m-1}^{(2)}=0, l_{m}^{(2)} \neq 0$. From (22) it follows that for an analytic two-parameter subgroup of $\Omega^{F}$ of class $m$, having the multiplication table of type $n(9)$, we have: $l_{0}^{(1)}=n / m$. In particular we have:

LEMMA 2. Let $F\left(z, a^{1}, a^{2}\right)$ be an analytic two-parameter (local or global) subgroup of $\Omega^{F}$ of class $n$, and hating the standard multiplication table of type $n$. Then $l_{0}^{(1)}=1$, $l_{0}^{(2)}=0$.

For every positive integer $n$ there actually exists a two-parameter analytic subgroup of $\Omega^{F}$ of the class $n$; for example:

$$
F\left(z, a^{1}, a^{2}\right)=\left(1+a^{1}\right) z /\left(1+a^{2} z^{n}\right)^{1 / n} .
$$

Indeed, for this group (which has the standard multiplication table of type $n$ ) we have

$$
L_{1}(z)=z, \quad L_{2}(z)=-z^{n+1} / n .
$$

3. Explicit form of the coefficients of an analytic two-parameter subgroup of $\Omega^{F}$. 3.1. Let

$$
F\left(z, a^{1}, a^{2}\right)=\sum_{q=1}^{\infty} f_{q}\left(a^{1}, a^{2}\right) z^{q}
$$

be an analytic two-parameter subgroup of $\Omega^{F}$. We propose to find an explicit expression for the coefficients $f_{q}\left(a^{1}, a^{2}\right)$, or, more generally, to find an explicit expression for the elements of the matrix $\left\|f_{m . q}\left(a^{1}, a^{2}\right)\right\|$ representing $F\left(z, a^{1}, a^{2}\right)$ (which contains the coefficients $f_{q}\left(a^{1}, a^{2}\right)$ as its first row).

The corresponding coefficients for the one-parameter analytic subgroups of $\Omega^{F}$ were obtained by E. Jabotinsky [6] for the simply connected subgroups, and by the author [8] for the nonsimply connected subgroups. It turned out, that in order to study the nonsimply connected subgroups, it was convenient to use a different multiplication table than the one used for the study of the simply connected subgroups.

In studying two-parameter subgroups of $\Omega^{F}$ we shall be led to use a different 
multiplication table for the study of each class of subgroups-namely, we shall use the standard multiplication table of type $n$ to study analytic two-parameter subgroups of $\Omega^{F}$ of class $n$.

Remembering that in every two-parameter analytic subgroup of $\Omega^{F}$ with essential parameters we can introduce in some neighborhood of the identity element a parametrization with the standard multiplication table (19) of type $n$, we can state the theorem:

THEOREM 2. Let $G$ be an analytic two-parameter (local or global) subgroup of $\Omega^{F}$ with essential parameters, and of class $n$. Let $D$ be a neighborhood of $\mathbf{a}=0$ in $C^{2}$ where the standard multiplication table of type $n$ can be introduced for some local subgroup $F\left(z, a^{1}, a^{2}\right)$ of $G$. Put

$$
\left(\frac{\partial F\left(z, a^{1}, a^{2}\right)}{\partial a^{i}}\right)_{\mathbf{a}=0}=\sum_{q=1}^{\infty} l_{q-1}^{(i)} z^{q}, \quad i=1,2 .
$$

Then, for $\left(a^{1}, a^{2}\right) \in D$, the elements of the matrix $\| f_{m, p}\left(a^{1}, a^{2}\right)$ which represents the group are given by:

$$
f_{m, p}\left(a^{1}, a^{2}\right)=\left(1+a^{1}\right)^{m} \delta_{m, p}, \quad p \leqq m
$$

and for $m<p$ :

$$
f_{m, p}\left(a^{1}, a^{2}\right)=\sum \frac{\left(a^{1}\right)^{s}}{s !} \frac{\left(a^{2}\right)^{r}}{r !} T_{s, j} \prod_{v=1}^{j} q_{v-1} l_{d_{v}}^{(1)} \prod_{\sigma=j+1}^{j+r} q_{\sigma-1} l_{d_{\sigma}}^{(2)},
$$

where the sum is taken over all integers $r, s: 0 \leqq s \leqq p, 0 \leqq r \leqq p-m, r+s>0$, and for every integer $j: 0 \leqq j \leqq s$, and all integers $q_{i}$ such that:

$$
m=q_{0} \leqq q_{1} \leqq \cdots \leqq q_{j}<q_{j+1}<\cdots<q_{j+r}=p
$$

and where $d_{i}=q_{i}-q_{i-1}$.

The $T_{s, j}$ are the Stirling numbers of the first kind, defined by the recurrence formulas:

$$
T_{0, j}=\delta_{0, j} \text { and } T_{s+1, j}=T_{s, j-1}-s T_{s, j}
$$

If the parameters $a^{1}, a^{2}$ are permitted to range through the set $\left\{\left(a^{1}, a^{2}\right) \mid a^{1} \neq-1\right\}$, formulas (23), (24) define a global two-parameter group $H$, which contains $G$ if $G$ was a local group, and coincides with $G$ if $G$ was global.

(Note. Further information about Stirling's numbers can be found in [7, Chapter IV].)

It is interesting to note, that formulas (23), (24) do not contain explicitly the number $n$, which is the class of $G$ and the type of the standard multiplication table used.

The proof of our Theorem 2 will be carried out in several steps. 
3.2. Let us introduce the following notation: given an analytic local or global two-parameter subgroup of $\Omega^{F}$

$$
F(z, \mathbf{a})=\sum_{q=1}^{\infty} f_{q}(\mathbf{a}) z^{q}
$$

we define, for every positive integer $t$ :

$$
[F(z, \mathbf{a})]^{t}=\sum_{q=0}^{\infty} f_{q}(\mathbf{a}, t) z^{q+t}=z^{t} \sum_{q=0}^{\infty} f_{q}(\mathbf{a}, t) z^{q} .
$$

Note that, $t$ being a positive integer, $f_{q}(\mathbf{a}, t)=f_{t, q+t}(\mathbf{a})$ where $f_{m, n}(\mathbf{a})$ is the appropriate element of the representation matrix. We also note, that if the series $F(z, a)$ has a nonzero radius of convergence for every a (that is, if $F(z, a)$ is a subgroup of $\Omega$ ), equation (26) is well defined for every complex $t$, with an appropriate choice of the branch. All the propositions that we are going to state about the coefficients $f_{q}(\mathbf{a}, t)$ remain valid for this case, too.

We first prove:

LEMMA 3. For an analytic two-parameter local subgroup of $\Omega^{F}, F\left(z, a^{1}, a^{2}\right)$ having the multiplication table $\phi(\mathbf{a}, \mathbf{b})$, and the tangent vectors:

we have

$$
\left(\frac{\partial F\left(z, a^{1}, a^{2}\right)}{\partial a^{i}}\right)_{\mathbf{a}=0}=\sum_{q=1}^{\infty} l_{q-1}^{(i)} z^{q}, \quad i=1,2,
$$

$$
f_{p}[\boldsymbol{\phi}(\mathbf{a}, \mathbf{b}), t]=\sum_{q=0}^{p} f_{q}(\mathbf{b}, t) f_{p-q}(\mathbf{a}, q+t),
$$

and also

$$
\left(\partial f_{q}(\mathbf{a}, t) / \partial a^{i}\right)_{\mathbf{a}=0}=t l_{q}^{(i)} .
$$

Proof. We rewrite the group property (5), raising both sides to the power $t$ :

or, using (26):

$$
\{F[z, \phi(\mathbf{a}, \mathbf{b})]\}^{t}=\{F[F(z, \mathbf{a}), \mathbf{b}]\}^{t}
$$

$$
\sum_{p=0}^{\infty} f_{p}[\boldsymbol{\phi}(\mathbf{a}, \mathbf{b}), t] z^{p+t}=\sum_{q=0}^{\infty} \sum_{s=0}^{\infty} f_{q}(\mathbf{b}, t) f_{s}(\mathbf{a}, q+t) z^{s+q+t}
$$

from which, by equating coefficients of powers of $z$, (27) follows.

To get (28), we differentiate (26) with respect to $a^{i}$, and put $\mathbf{a}=\mathbf{0}$. As $F(z, 0)=z$ we get:

$$
t z^{t-1} \sum_{q=0}^{\infty} l_{q-1}^{(i)} z^{q}=\sum_{q=0}^{\infty}\left(\frac{\partial f_{q}(\mathbf{a}, t)}{\partial a^{i}}\right)_{\mathbf{a}=0} z^{q+t}
$$

from which (28) follows.

3.3. We now prove 
LEMMA 4. Let the analytic two-parameter local or global subgroup of $\Omega^{F}$, $F\left(z, a^{1}, a^{2}\right)$, be of class $n$ and be written in the standard multiplication table of type $n$. Then the corresponding coefficients $f_{q}\left(a^{1}, a^{2}, t\right)$ have the form:

$$
f_{q}\left(a^{1}, a^{2}, t\right)=\left(1+a^{1}\right)^{t} \cdot P_{q}\left(a^{1}, a^{2}, t\right)
$$

where $P_{q}\left(a^{1}, a^{2}, t\right)$ are polynomials of degree not exceeding $q$ in each of the parameters $a^{1}$ and $a^{2}$.

Proof. I. We first seek the explicit expression for $f_{0}\left(a^{1}, a^{2}, t\right)$. Equation (27), written for standard multiplication table of type $n$ and for $p=0$ becomes

$$
f_{0}\left[a^{1}+b^{1}+a^{1} b^{1}, a^{2}+b^{2}\left(1+a^{1}\right)^{n}, t\right]=f_{0}\left(b^{1}, b^{2}, t\right) f_{0}\left(a^{1}, a^{2}, t\right) .
$$

Differentiating with respect to $a^{2}$ and putting $\mathbf{a}=\mathbf{0}$ we get, using (28):

$$
\partial f_{0}\left(b^{1}, b^{2}, t\right) / \partial b^{2}=f_{0}\left(b^{1}, b^{2}, t\right) l_{0}^{(2)} t \text {. }
$$

As by Lemma $2: l_{0}^{(2)}=0$, it follows from $(31)$ that $f_{0}\left(b^{1}, b^{2}, t\right)$ is actually independent of $b^{2}$.

Differentiating now (30) with respect to $b^{1}$, and putting $\mathbf{b}=\mathbf{0}$, we get, using (28):

$$
\left(1+a^{1}\right) \partial f_{0}\left(a^{1}, a^{2}, t\right) / \partial a^{1}=t l_{0}^{(1)} f_{0}\left(a^{1}, a^{2}, t\right)
$$

which implies, as $f_{0}\left(a^{1}, a^{2}, t\right)$ is independent of $a^{2}$,

$$
f_{0}\left(a^{1}, a^{2}, t\right)=\left(1+a^{1}\right)^{l_{0}^{(1)} t} K(t) .
$$

Putting $\mathbf{a}=\mathbf{0}$ and noting that $f_{0}(0,0, t)=1$ we find $k(t) \equiv 1$. By Lemma 2 we have $l_{0}^{(1)}=1$, so that, finally:

$$
f_{0}\left(a^{1}, a^{2}, t\right)=\left(1+a^{1}\right)^{t}
$$

The lemma is thus valid for $q=0$.

II. We proceed, by induction on $q$, to show that $f_{q}\left(a^{1}, a^{2}, t\right)$ is a polynomial in $a^{2}$ of degree not exceeding $q$. The case $q=0$ is covered already; suppose that so is the case for $q=0,1, \ldots, p-1$.

Differentiate (27), where $\phi(\mathbf{a}, \mathbf{b})$ is taken to be the standard multiplication table of type $n$, with respect to $a^{2}$, and put $\mathbf{a}=0$. Because of (28) we get

$$
\frac{\partial f_{p}\left(b^{1}, b^{2}, t\right)}{\partial b^{2}}=\sum_{q=0}^{p-1} f_{q}\left(b^{1}, b^{2}, t\right) l_{p-q}^{(2)}(q+t) .
$$

(The summation goes only up to $q=p-1$, because $l_{0}^{(2)}=0$.) By the induction hypothesis, each term in the sum is a polynomial of degree not exceeding $p-1$ in $b^{2}$; hence $f_{p}\left(b^{1}, b^{2}, t\right)$ is a polynomial of degree not exceeding $p$ in $b^{2}$. Hence, in (29) $P_{q}\left(a^{1}, a^{2}, t\right)$, qua function of $a^{2}$, is a polynomial of degree not exceeding $q$.

III. It remains to prove that $P_{q}\left(a^{1}, a^{2}, t\right)$, qua function of $a^{1}$, is also a polynomial of degree not exceeding $q$. We shall complete the proof again by induction on $q$. Equation (33) proves the statement for $q=0$. Suppose that the statement 
holds for $q=0,1, \ldots, p-1$. Differentiate $(27)$, where $\boldsymbol{\phi}(\mathbf{a}, \mathbf{b})$ is taken to be the standard multiplication table of type $n$, with respect to $b^{1}$ and put $\mathbf{b}=\mathbf{0}$; because of (28) we get

$$
\left(1+a^{1}\right) \frac{\partial f_{p}\left(a^{1}, a^{2}, t\right)}{\partial a^{1}}=\sum_{q=0}^{p} t l_{q}^{(1)} f_{p-q}\left(a^{1}, a^{2}, q+t\right) .
$$

Transfer to the left the term which contains $f_{p}\left(a^{1}, a^{2}, t\right)$, remembering that, by Lemma $2, l_{0}^{(1)}=1$. Applying the induction hypothesis to the sum on the right, we get

$$
\begin{aligned}
\left(1+a^{1}\right) \frac{\partial f_{p}\left(a^{1}, a^{2}, t\right)}{\partial a^{1}}-t f_{p} & \left(a^{1}, a^{2}, t\right) \\
= & \left(1+a^{1}\right)^{t+1} \sum_{q=1}^{p} t l_{q}^{(1)} P_{p-q}\left(a^{1}, a^{2}, q+t\right)\left(1+a^{1}\right)^{q-1}
\end{aligned}
$$

where $P_{p-q}\left(a^{1}, a^{2}, q+t\right)$ is a polynomial in $a^{1}$ of degree not exceeding $p-q$. The last equation can be rewritten, after dividing both sides by $\left(1+a^{1}\right)^{t+1}$, in the form:

$$
\frac{\partial}{\partial a^{1}}\left\{\frac{f_{p}\left(a^{1}, a^{2}, t\right)}{\left(1+a^{1}\right)^{t}}\right\}=\sum_{q=1}^{p} t l_{q}^{(1)} P_{p-q}\left(a^{1}, a^{2}, q+t\right)\left(1+a^{1}\right)^{q-1}
$$

hence, using the induction hypothesis, we find that

$$
P_{p}\left(a^{1}, a^{2}, t\right)=f_{p}\left(a^{1}, a^{2}, t\right) /\left(1+a^{1}\right)^{t}
$$

is a polynomial of degree not exceeding $p$ in $a^{1}$; this completes the proof of the lemma.

3.4. We now state:

LEMMA 5. Let $F\left(z, a^{1}, a^{2}\right)$ be a local or global analytic two-parameter subgroup of $\Omega^{F}$, with a standard multiplication table of arbitrary type. Then the derivatives of $f_{p}\left(a^{1}, a^{2}, t\right)$ with respect to $a^{1}$ are given by:

$$
\begin{aligned}
& \frac{\partial^{s} f_{p}\left(a^{1}, a^{2}, t\right)}{\partial\left(a^{1}\right)^{s}} \\
& \quad=\frac{1}{\left(1+a^{1}\right)^{s}} \sum T_{s, j} \prod_{v=1}^{j}\left(q_{v-1}+t\right) l_{d_{v}}^{(1)} f_{p-q_{j}}\left(a^{1}, a^{2}, q_{j}+t\right) \quad(s \geqq 1)
\end{aligned}
$$

where the sum is taken for every integer $j: 1 \leqq j \leqq s$ and for all integers $q_{i}$ such that: $0=q_{0} \leqq q_{1} \leqq \cdots \leqq q_{j} \leqq p$ and where $d_{v}=q_{v}-q_{v-1}$; and the derivatives of $f_{p}\left(a^{1}, a^{2}, t\right)$ with respect to $a^{2}$ are given by

$$
\frac{\partial^{r} f_{p}\left(a^{1}, a^{2}, t\right)}{\partial\left(a^{2}\right)^{r}}=\sum \prod_{\sigma=1}^{r}\left(k_{\sigma}+t\right) l_{d_{\sigma}}^{(2)} f_{k_{r}}\left(a^{1}, a^{2}, t\right) \quad(r \geqq 1)
$$

where the sum is taken for all integers $k_{i}$ such that

$$
0 \leqq k_{r}<k_{r-1}<\cdots<k_{1}<k_{0}=p,
$$

and where $e_{\sigma}=k_{\sigma-1}-k_{\sigma}$. 
Proof. (I) We shall first prove (34) by induction on s. Differentiate (27), where $\boldsymbol{\phi}(\mathbf{a}, \mathbf{b})$ is taken to be any standard multiplication table of the form (19), with respect to $b^{1}$ and put $\mathrm{b}=0$; because of (28) we get

$$
\left(1+a^{1}\right) \frac{\partial f_{p}\left(a^{1}, a^{2}, t\right)}{\partial a^{1}}=\sum_{q=0}^{p} t l_{q}^{(1)} f_{p-q}\left(a^{1}, a^{2}, q+t\right)
$$

which is (34) for the case $s=1$ (because $T_{1,1}=1$ ).

Assuming (34) to hold for the derivative of order $s$, we differentiate it to compute the derivative of order $s+1$ :

$$
\begin{aligned}
\frac{\partial^{s+1} f_{p}\left(a^{1}, a^{2}, t\right)}{\partial\left(a^{1}\right)^{s+1}}= & \frac{1}{\left(1+a^{1}\right)^{s}} \sum T_{s, j} \prod_{v=1}^{j}\left(q_{v-1}+t\right) l_{d_{v}}^{(1)} \frac{\partial}{\partial a^{1}}\left[f_{p-q_{j}}\left(a^{1}, a^{2}, q_{j}+t\right)\right] \\
& -\frac{s}{\left(1+a^{1}\right)^{s+1}} \sum T_{s, j} \prod_{v=1}^{j}\left(q_{v-1}+t\right) l_{d_{v}}^{(1)} f_{p-q_{j}}\left(a^{1}, a^{2}, q_{j}+t\right)
\end{aligned}
$$

where the sums are taken as in (34). By (36), we have

$$
\left(1+a^{1}\right) \frac{\partial}{\partial a^{1}}\left[f_{p-q_{j}}\left(a^{1}, a^{2}, q_{j}+t\right)\right]=\sum_{q=0}^{p-q_{j}}\left(q_{j}+t\right) l_{q}^{(1)} f_{p-q_{j}-q}\left(a^{1}, a^{2}, q+q_{j}+t\right) .
$$

In the last sum we put $q_{j+1}=q_{j}+q$, so that $q_{j} \leqq q_{j+1} \leqq p$. Substituting (37) with the new index $q_{j+1}$ into the first sum of the expression for the $(s+1)$ th derivative, we get

$$
\begin{aligned}
\frac{\partial^{s+1} f_{p}\left(a^{1}, a^{2}, t\right)}{\partial\left(a^{1}\right)^{s+1}}= & \frac{1}{\left(1+a^{1}\right)^{s+1}} \sum T_{s, j} \prod_{v=1}^{j+1}\left(q_{v-1}+t\right) l_{d_{v}}^{(1)} f_{p-q_{j+1}}\left(a^{1}, a^{2}, q_{j+1}+t\right) \\
& -\frac{1}{\left(1+a^{1}\right)^{s+1}} \sum s T_{s, j} \prod_{v=1}^{j}\left(q_{v-1}+t\right) l_{d_{v}}^{(1)} f_{p-q_{j}}\left(a^{1}, a^{2}, q_{j}+t\right)
\end{aligned}
$$

where the first sum is taken for every $j: 1 \leqq j \leqq s$ and for all integers $q_{i}$ such that: $0 \leqq q_{0} \leqq q_{1} \leqq \cdots \leqq q_{j} \leqq q_{j+1} \leqq p$ and where $d_{v}=q_{v}-q_{\nu-1}$; the second sum is taken as in (34).

In the first sum we replace $j$ by $j-1$ and remember that $T_{s, 0}=0$. In the second sum we allow $j$ to range from 1 to $s+1$, as $T_{s, s+1}=0$. The expression for the $(s+1)$ th derivative becomes:

$$
\begin{aligned}
\frac{\partial^{s+1} f_{p}\left(a^{1}, a^{2}, t\right)}{\partial\left(a^{1}\right)^{s+1}}= & \frac{1}{\left(1+a^{1}\right)^{s+1}} \sum T_{s, j-1} \prod_{v=1}^{j}\left(q_{v-1}+t\right) l_{d_{v}}^{(1)} f_{p-q_{j}}\left(a^{1}, a^{2}, q_{j}+t\right) \\
& -\frac{1}{\left(1+a^{1}\right)^{s+1}} \sum s T_{s, j} \prod_{v=1}^{j}\left(q_{v-1}+t\right) l_{d_{v}}^{(1)} f_{p-q}\left(a^{1}, a^{2}, q_{j}+t\right)
\end{aligned}
$$

where the sums are taken for all integers $j: 1 \leqq j \leqq s+1$ and all integers $q_{i}$ such that: $0 \leqq q_{0} \leqq q_{1} \leqq \cdots \leqq q_{j-1} \leqq q_{j} \leqq p$ and where $d_{v}=q_{v}-q_{v-1}$.

Finally, using (25), we get

$$
\frac{\partial^{s+1} f_{p}\left(a^{1}, a^{2}, t\right)}{\partial\left(a^{1}\right)^{s+1}}=\frac{1}{\left(1+a^{1}\right)^{s+1}} \sum T_{s+1, j} \prod_{v=1}^{j}\left(q_{v-1}+t\right) l_{d_{v}}^{(1)} f_{p-q_{j}}\left(a^{1}, a^{2}, q_{j}+t\right)
$$


where the sum is taken as before. This is the statement of the lemma for the $(s+1)$ th derivative with respect to $a^{1}$, and formula (34) is proved.

(II) We shall prove now (35) by induction on $r$. Differentiate (27), where $\phi(\mathbf{a}, \mathbf{b})$ is taken to be any standard multiplication table of the form (19), with respect to $a^{2}$ and put $\mathbf{a}=\mathbf{0}$; we get, after replacing $\mathbf{b}$ by $\mathbf{a}$ :

$$
\frac{\partial f_{p}\left(a^{1}, a^{2}, t\right)}{\partial a^{2}}=\sum_{q=0}^{p-1}(q+t) l_{p-q}^{(2)} f_{q}\left(a^{1}, a^{2}, t\right) .
$$

(The summation is only up to $q=p-1$ because $l_{0}^{(2)}=0$.) Formula (38) coincides with (35) for $r=1$. Assume (35) to be valid for the $r$ th derivative. Differentiation of (35) with respect to $a^{2}$ yields

$$
\frac{\partial^{r+1} f_{p}\left(a^{1}, a^{2}, t\right)}{\partial\left(a^{2}\right)^{r+1}}=\prod_{\sigma=1}^{r}\left(k_{\sigma}+t\right) l_{e_{\sigma}}^{(2)} \frac{\partial}{\partial a^{2}} f_{k_{1}}\left(a^{1}, a^{2}, t\right)
$$

where the sum is taken as in (35). Applying (38) we get

$$
\frac{\partial^{r+1} f_{p}\left(a^{1}, a^{2}, t\right)}{\partial\left(a^{2}\right)^{r+1}}=\sum \prod_{\sigma=1}^{r}\left(k_{\sigma}+t\right) l_{e_{\sigma}}^{(2)} \sum_{k_{r+1}=0}^{k_{r}-1}\left(k_{r+1}+t\right) l_{k_{r}-k_{r+1}}^{(2)} f_{k_{r+1}}\left(a^{1}, a^{2}, t\right)
$$

where the sums are taken as in (35); the last equality may be rewritten in the form

$$
\frac{\partial^{r+1} f_{p}\left(a^{1}, a^{2}, t\right)}{\partial\left(a^{2}\right)^{r+1}}=\sum \prod_{\sigma=1}^{r+1}\left(k_{\sigma}+t\right) l_{e_{\sigma}}^{(2)} f_{k_{r+1}}\left(a^{1}, a^{2}, t\right)
$$

where the sum is taken for all positive integers $k_{i}$ such that $0 \leqq k_{r+1}<k_{r}<\cdots<k_{0}=p$ and where $e_{\sigma}=k_{\sigma-1}-k_{\sigma}$. As this is the statement of the lemma for the $(s+1)$ th derivative with respect to $a^{2}$, the proof is complete.

3.5. We can now proceed to obtain the Maclaurin expansion of $f_{p}\left(a^{1}, a^{2}, t\right)$. Combining the formulas (34) and (35), we obtain:

$$
\begin{aligned}
& \frac{\partial^{s+r} f_{p}\left(a^{1}, a^{2}, t\right)}{\partial\left(a^{1}\right)^{s} \partial\left(a^{2}\right)^{r}} \\
& =\frac{1}{\left(1+a^{1}\right)^{s}} \sum T_{s, j} \prod_{v=1}^{j}\left(q_{v-1}+t\right) l_{d_{v}}^{(1)} \prod_{\sigma=1}^{r}\left(k_{\sigma}+t\right) l_{e_{\sigma}}^{(2)} f_{k_{r}-q_{j}}\left(a^{1}, a^{2}, q_{j}+t\right) \\
& \quad(s+r>0)
\end{aligned}
$$

where the sum is taken for all integers $j: 0 \leqq j \leqq s$ and for all integers $q_{i}$ and $k_{i}$ such that:

$$
0=q_{0} \leqq q_{1} \leqq \cdots \leqq q_{j} \leqq k_{r}<k_{r-1}<\cdots<k_{1}<k_{0}=p
$$

and where $d_{v}=q_{v}-q_{v-1}, e_{\sigma}=k_{\sigma-1}-k_{\sigma}\left(\right.$ and $\left.\prod_{v=1}^{0} A_{v}=1\right)$.

In (39) put $\mathbf{a}=\mathbf{0}$. The only terms which do not vanish are those for which $k_{r}=q_{j}$. Noting that $f_{0}\left(0,0, q_{j}+t\right)=1$, we get

$$
\frac{\partial^{s+r} f_{p}(0,0, t)}{\partial\left(a^{1}\right)^{s} \partial\left(a^{2}\right)^{r}}=\sum T_{s, j} \prod_{v=1}^{j}\left(q_{v-1}+t\right) l_{d_{v}}^{(1)} \prod_{\sigma=1}^{r}\left(k_{\sigma}+t\right) l_{e_{\sigma}}^{(2)}
$$


where the sum is taken for all integers $j: 0 \leqq j \leqq s$ and for all integers $q_{i}$ and $k_{i}$ such that

$$
0 \leqq q_{0} \leqq q_{1} \leqq \cdots \leqq q_{j}=k_{r}<k_{r-1}<\cdots<k_{1}<k_{0}=p
$$

and where $d_{v}=q_{v}-q_{v-1}, e_{\sigma}=k_{\sigma-1}-k_{\sigma}$.

Renaming the integers $k_{\alpha}, \alpha=0,1, \ldots, r$ by writing $k_{\alpha}=q_{j+r-\alpha}$ we obtain (40) in the form

$$
\frac{\partial^{s+r} f_{p}(0,0, t)}{\partial\left(a^{1}\right)^{s} \partial\left(a^{2}\right)^{r}}=\sum T_{s, j} \prod_{v=1}^{j}\left(q_{v-1}+t\right) l_{d_{v}}^{(1)} \prod_{\sigma=j+1}^{r+j}\left(q_{\sigma-1}+t\right) l_{d_{\sigma}}^{(2)}
$$

where the sum is taken for every integer $j: 0 \leqq j \leqq s$, and for all integers $q_{i}$ such that:

$$
0 \leqq q_{0} \leqq q_{1} \leqq \cdots \leqq q_{j}<q_{j+1}<\cdots<q_{j+r}=p
$$

and where $d_{i}=q_{i}-q_{i-1}$.

Using this, equation (32), and, for $p>0$ Maclaurin's formula:

$$
f_{p}\left(a^{1}, a^{2}, t\right)=\sum_{s, r=0}^{\infty} \frac{\left(a^{1}\right)^{s}}{s !} \frac{\left(a^{2}\right)^{r}}{r !} \frac{\partial^{s+r} f_{p}(0,0, t)}{\partial\left(a^{1}\right)^{s} \partial\left(a^{2}\right)^{r}}
$$

(noting that $f_{p}(0,0, t)=0$ for $\left.p>0\right)$ we obtain:

LEMMA 6. Let $F\left(z, a^{1}, a^{2}\right)$ be a local or global analytic two-parameter subgroup of $\Omega^{F}$, with a standard multiplication table of arbitrary type. Then the coefficients $f_{q}\left(a^{1}, a^{2}, t\right)$ are given by

$$
f_{0}\left(a^{1}, a^{2}, t\right)=\left(1+a^{1}\right)^{l_{0}^{(1)} t}
$$

and for $p \geqq 1$ :

$$
f_{p}\left(a^{1}, a^{2}, t\right)=\sum \frac{\left(a^{1}\right)^{s}}{s !} \frac{\left(a^{2}\right)^{r}}{r !} T_{s, j} \prod_{v=1}^{j}\left(q_{v-1}+t\right) l_{d_{v}}^{(1)} \prod_{\sigma=j+1}^{r+j}\left(q_{\sigma-1}+t\right) l_{d_{\sigma}}^{(2)}
$$

where the sum is taken over all integers $r, s: 0 \leqq s, r<\infty, s+r>0$, and for every integer $j: 0 \leqq j \leqq s$ and for all integers $q_{i}$ such that

$$
0=q_{0} \leqq q_{1} \leqq \cdots \leqq q_{j}<q_{j+1}<\cdots<q_{j+r}=p
$$

and where $d_{i}=q_{i}-q_{t-1}$.

We note that Lemma 6 holds for all integer values of $t$, and when $F\left(z, a^{1}, a^{2}\right)$ is a subgroup of $\Omega$-for all complex values of $t$, with the appropriate choice of the branch for $f_{q}\left(a^{1}, a^{2}, t\right)$ in (26). We shall use Lemma 6 only for the case where $t$ is a positive integer, but it can be used to yield additional information about the subgroup, as the elements of the generalized representation matrix $\left\|f_{m, q}\left(a^{1}, a^{2}\right)\right\|$, $-\infty<m, q<\infty$, defined by:

(see [5]).

$$
\left[F\left(z, a^{1}, a^{2}\right)\right]^{m}=\sum_{q=-\infty}^{\infty} f_{m . q}\left(a^{1}, a^{2}\right) z^{q}
$$


3.6. Proof of Theorem 2. Using the relations $f_{m, p}\left(a^{1}, a^{2}\right)=f_{p-m}\left(a^{1}, a^{2}, m\right)$ for $p \geqq m$ and $f_{m, p}\left(a^{1}, a^{2}\right)=0$ for $p<m$ we get from Lemma 6 :

$$
f_{m, p}\left(a^{1}, a^{2}\right)=\left(1+a^{1}\right)^{l_{0}^{(1)} m} \cdot \delta_{m, p} \text { for } p \leqq m,
$$

and for $p>m$ :

$$
f_{m, p}\left(a^{1}, a^{2}\right)=\sum \frac{\left(a^{1}\right)^{s}}{s !} \frac{\left(a^{2}\right)^{r}}{r !} T_{s, j} \prod_{v=1}^{j}\left(q_{v-1}+m\right) l_{d_{v}}^{(1)} \prod_{\sigma=j+1}^{r+j}\left(q_{\sigma-1}+m\right) l_{d_{\sigma}}^{(2)}
$$

where the sum is taken over all integers $r, s: 0 \leqq r, s<\infty, r+s>0$, and over all integers $j: 0 \leqq j \leqq s$, and for all integers $q_{i}$ such that

$$
0 \leqq q_{0} \leqq q_{1} \leqq \cdots \leqq q_{j}<q_{j+1}<\cdots<q_{j+r}=p-m
$$

and where $d_{i}=q_{i}-q_{i-1}$.

This is valid for a subgroup of any class, written in the standard multiplication table of any type. For groups of class $n$ written in the standard multiplication table of type $n$ we have, by Lemma $2, l_{0}^{(1)}=1$. Formula (23) in the statement of Theorem 2 now follows from (44). From Lemma 4 it follows, that for such a group, $f_{p-m}\left(a^{1}, a^{2}, m\right)$ which is $f_{m, p}\left(a^{1}, a^{2}\right)$, will be a polynomial of degree not exceeding $p$ in $a^{1}$, and of degree $p-m$ at most in $a^{2}$; hence, in (45), the summations over $s$ and $r$ only include terms for $0 \leqq s \leqq p$ and $0 \leqq r \leqq p-m$, yielding (24).

(It is interesting to remark that we have hereby shown that the summation figuring in (45) taken for a fixed value of $s$, such that $s>p$, vanish. This constitutes a combinatorial theorem already noted by the author [8], and for which a combinatorial proof was given by $\mathrm{H}$. Hanani [4].)

It remains to prove that when the parameter $a$ is permitted to range in the domain $\left\{\left(a^{1}, a^{2}\right) \mid a^{1} \neq-1\right\}$ the coefficients $f_{m, p}\left(a^{1}, a^{2}\right)$ given by (23) and (24) define a global analytic two-parameter subgroup of $\Omega^{F}$. From (23) follows, that as long as $a^{1} \neq-1, f_{1}\left(a^{1}, a^{2}\right) \neq 0$, and hence the formal power series:

$$
F\left(z, a^{1}, a^{2}\right)=\sum_{q=1}^{\infty} f_{q}\left(a^{1}, a^{2}\right) z^{q}=\sum_{q=1}^{\infty} f_{1, q}\left(a^{1}, a^{2}\right) z^{q}
$$

belongs to $\Omega^{F}$. The coefficients $f_{q}\left(a^{1}, a^{2}\right)$ are, for small values of $a^{1}$ and $a^{2}$, the coefficients of the local group from which we started in the statement of Theorem 2 ; hence $f_{q}\left(a^{1}, a^{2}\right)$ satisfy, in some neighborhood of $\mathbf{a}=\mathbf{0}$ the relations which follow from

$$
F[F(z, \mathbf{a}), \mathbf{b}]=F\left[z, \phi_{n}(\mathbf{a}, \mathbf{b})\right]
$$

But $f_{q}\left(a^{1}, a^{2}\right)$, being polynomials satisfying a set of functional equations on an open set, satisfy these equations for all values of the parameters, and hence (46) holds whenever $\mathbf{a}, \mathbf{b} \in\left\{\left(a^{1}, a^{2}\right) \mid a^{1} \neq-1\right\}$, and therefore $F\left(z, a^{1}, a^{2}\right)$ is a global group. This finishes the proof of Theorem 2 . 
4. Canonical representation of two-parameter analytic subgroups of $\Omega^{F}$.

4.1. It is our purpose to prove:

THEOREM 3. Every two-parameter analytic subgroup of $\Omega^{F}$ of class $n$ has a representation of the form

$$
F\left(z, a^{1}, a^{2}\right)=\phi^{-1}\left[\left(1+a^{1}\right) \cdot \phi(z) /\left(1+a^{2}[\phi(z)]^{n}\right)^{1 / n}\right]
$$

where $\phi(z)=\sum_{q=1}^{\infty} \phi_{q} z^{q} \in \Omega^{F}$ and $\phi_{1}=1$.

Moreover,

$$
g\left(z, a^{1 *}, a^{2 *}\right)=\psi^{-1}\left[\left(1+a^{1 *}\right) \cdot \psi(z) /\left(1+a^{2 *}[\psi(z)]^{n}\right)^{1 / n}\right],
$$

where $\psi(z)=\sum_{q=1}^{\infty} \psi_{q} z^{q} \in \Omega^{F}$ and $\psi_{1}=1$, is another representation of the subgroup (47) if and only if

$$
\psi(z)=\phi(z) /\left(1+k[\phi(z)]^{n}\right)^{1 / n}
$$

for some complex $k$.

4.2. In order to prove Theorem 3, we first prove:

LEMMA 7. Let a formal power series $L_{1}(z)=z+\sum_{q=2}^{\infty} l_{q-1}^{(1)} z^{q}$ and a positive integer $n$ be given.

Define the formal power series $\Lambda(z)$ by

$$
1 / L_{1}(z)=1 / z+\Lambda(z)
$$

Further, define the formal power series $\phi(z) \in \Omega^{F}$ by

$$
\phi(z)=z \exp \left[\int_{0}^{z} \Lambda(z) d z\right]
$$

Then, the two-parameter analytic subgroup of $\Omega^{F}$ of class $n$ (written in the standard multiplication table of type $n$ ), defined by

$$
F\left(z, a^{1}, a^{2}\right)=\phi^{-1}\left[\left(1+a^{1}\right) \cdot \phi(z) /\left(1+a^{2}[\phi(z)]^{n}\right)^{1 / n}\right]
$$

satisfies

$$
\left(\partial F\left(z, a^{1}, a^{2}\right) / \partial a^{1}\right)_{\mathbf{a}=0}=L_{1}(z) .
$$

Proof. Differentiating (52) with respect to $a^{1}$ and putting $\mathrm{a}=0$ we get

$$
\left(\partial F\left(z, a^{1}, a^{2}\right) / \partial a^{1}\right)_{\mathbf{a}=0}=\phi^{-1^{\prime}}[\phi(z)] \cdot \phi(z) .
$$

Using the identity $\phi^{-1^{\prime}}[\phi(z)]=1 / \phi^{\prime}(z)$, and $\phi^{\prime}(z)=\phi(z) / z+\phi(z) \cdot \Lambda(z)$ (obtained from (51)), (54) yields (53), by use of (50).

4.3. We have furthermore:

LEMMA 8. Let a formal power series $L_{1}(z)=z+\sum_{q=2}^{\infty} l_{q-1}^{(1)} z^{q}$ and a positive integer $n$ be given. Then there exists a unique two-parameter analytic subgroup of $\Omega^{F}$ of class $n, F\left(z, a^{1}, a^{2}\right)$, written in the standard multiplication table of type $n$, such that

$$
\left(\partial F\left(z, a^{1}, a^{2}\right) / \partial a^{1}\right)_{\mathbf{a}=0}=L_{1}(z),
$$


and $L_{2}(z)$ starts with the term $-z^{n+1} / n$, that is,

$$
L_{2}(z)=\left(\frac{\partial F\left(z, a^{1}, a^{2}\right)}{\partial a^{2}}\right)_{a=0}=-\frac{1}{n} z^{n+1}+\sum_{q=n+2}^{\infty} l_{q-1}^{(2)} z^{q}
$$

Proof. Noting that the group given by (52) satisfies (56), the existence of the required group is covered by Lemma 7 . It remains to show that $n, L_{1}(z)$ and the condition (56) determine the group completely. The integrability condition (20) for an analytic two-parameter subgroup of $\Omega^{F}$ written in the standard multiplication table of type $n$ shows us that $L_{1}(z), n$, and the condition (56) determine $L_{2}(z)$ completely. As by Theorem $2 F\left(z, a^{1}, a^{2}\right)$ is completely determined by $L_{1}(z)$ and $L_{2}(z)$, the lemma is proved.

4.4. Proof of Theorem 3. (I) Let an analytic two-parameter subgroup of $\Omega^{F}$ of class $n, F\left(z, a^{1}, a^{2}\right)$, be given. We may assume that the parametrization is written in the standard multiplication table of type $n$.

We note, that a transformation of the group parameters of the form $a^{1}=a^{1 *}$, $a^{2}=k a^{2 *}$, where $k \neq 0$ is some fixed complex number, leaves the standard multiplication table of type $n$ unchanged, and we have

$$
L_{2}^{*}(z)=\left(\frac{\partial F^{*}\left(z, a^{1 *}, a^{2 *}\right)}{\partial a^{2 *}}\right)_{\mathbf{a}^{*}=0}=\left(\frac{\partial F\left(z, a^{1 *}, k a^{2 *}\right)}{\partial a^{2 *}}\right)_{\mathbf{a}^{*}=0}=k L_{2}(z)
$$

It follows, that $k$ may be so chosen, that condition (56) is satisfied (that is, so that the coefficient of the first term in $L_{2}^{*}(z)$ will be $\left.-1 / n\right)$. Hence we may assume that $F\left(z, a^{1}, a^{2}\right)$ is written in the standard multiplication table of type $n$ and that condition (56) holds.

By Lemma 2 we have

$$
L_{1}(z)=\left(\frac{\partial F\left(z, a^{1}, a^{2}\right)}{\partial a^{1}}\right)_{\mathbf{a}=0}=z+\sum_{q=2}^{\infty} l_{q-1}^{(1)} z^{q}
$$

and, using Lemma 7, we can construct a group having the representation (52) and satisfying (53); by Lemma 8 the constructed group coincides with the subgroup from which we have started, and we have shown that the given subgroup, being of class $n$, has a representation of the form (47).

(II) Suppose now that a two-parameter analytic subgroup of $\Omega^{F}$ is represented by (47), and $\psi(z)$ is given by (49). We shall see, that $g\left(z, a^{1 *}, a^{2 *}\right)$ given by (48) is another representation of the same group.

Write (49) in the form

$$
\psi(z)=\frac{z}{\left(1+k z^{n}\right)^{1 / n}} \circ \phi(z)
$$

it follows that

$$
\psi^{-1}(z)=\phi^{-1}(z) \circ z /\left(1-k z^{n}\right)^{1 / n}
$$


Introducing (57) and (58) into (48) we get

$$
\begin{aligned}
g\left(z, a^{1 *}, a^{2 *}\right) & =\phi^{-1}(z) \circ \frac{z}{\left(1-k z^{n}\right)^{1 / n}} \circ \frac{\left(1+a^{1 *}\right) z}{\left(1+a^{2 *} z^{n}\right)^{1 / n}} \circ \frac{z}{\left(1+k z^{n}\right)^{1 / n}} \circ \phi(z) \\
& =\phi^{-1}\left[\frac{\left(1+a^{1 *}\right) \cdot \phi(z)}{\left(1+\left\{a^{2 *}+k-k\left(1+a^{1 *}\right)^{n}\right\}[\phi(z)]^{n}\right)^{1 / n}}\right]
\end{aligned}
$$

or

$$
g\left(z, a^{1 *}, a^{2 *}\right)=\phi^{-1}\left[\left(1+a^{1}\right) \cdot \phi(z) /\left(1+a^{2}[\phi(z)]^{n}\right)^{1 / n}\right]
$$

where $a^{1}=a^{1 *}$ and $a^{2}=a^{2 *}+k-k\left(1+a^{1 *}\right)^{n}$.

When $\left(a^{1 *}, a^{2 *}\right)$ ranges in the domain $\left\{\left(a^{1 *}, a^{2 *}\right) \mid a^{1 *} \neq-1\right\}$ the elements of the subgroup $g\left(z, a^{1 *}, a^{2 *}\right)$ are all the elements of $F\left(z, a^{1}, a^{2}\right)$, hence $g\left(z, a^{1}, a^{2}\right)$ is another representation of the subgroup $F\left(z, a^{1}, a^{2}\right)$ given by (47).

(III) Suppose now that (47) and (48) are different representations of the same subgroup, that is, there exist functions $\theta_{1}\left(a^{1}, a^{2}\right)$ and $\theta_{2}\left(a^{1}, a^{2}\right)$ such that:

$$
\phi^{-1}\left[\frac{\left(1+a^{1}\right) \cdot \phi(z)}{\left(1+a^{2}[\phi(z)]^{n}\right)^{1 / n}}\right]=\psi^{-1}\left[\frac{\left\{1+\theta_{1}\left(a^{1}, a^{2}\right)\right\} \psi(z)}{\left(1+\theta_{2}\left(a^{1}, a^{2}\right)[\psi(z)]^{n}\right)^{1 / n}}\right]
$$

Comparing coefficients of $z$ on both sides of (59) we get $\theta_{1}\left(a^{1}, a^{2}\right)=a^{1}$. Put $a^{1}=0$ in (59). We get

$$
\phi^{-1}\left[\frac{\phi(z)}{\left(1+a^{2}[\phi(z)]^{n}\right)^{1 / n}}\right]=\psi^{-1}\left[\frac{\psi(z)}{\left(1+\theta_{2}\left(0, a^{2}\right)[\psi(z)]^{n}\right)^{1 / n}}\right]
$$

Differentiate (60) with respect to $a^{2}$ and put $a^{2}=0$. Noting that $\theta_{2}(0,0)=0$ follows from (60), we get

$$
-\frac{1}{n} \frac{[\phi(z)]^{n+1}}{\phi^{\prime}(z)}=-\frac{1}{n} \frac{[\psi(z)]^{n+1}}{\psi^{\prime}(z)} \cdot \frac{\partial \theta_{2}(0,0)}{\partial a^{2}} .
$$

Comparing the coefficients of $z^{n+1}$ on both sides we get

$$
\partial \theta_{2}(0,0) / \partial a^{2}=1
$$

Formal integration of (61) yields

$$
1 /[\phi(z)]^{n}+k=1 /[\psi(z)]^{n}
$$

from which (49) follows, and the proof of Theorem 3 is complete.

4.5. From Theorem 3 it follows, that every two-parameter analytic subgroup of $\Omega^{F}$ of class $n$ is globally isomorphic to the group:

$$
H_{n}\left(z, a^{1}, a^{2}\right)=\left(1+a^{1}\right) z /\left(1+a^{2} z^{n}\right)^{1 / n} .
$$

As $H_{n}\left(z, a^{1}, a^{2}\right)$ is not globally isomorphic to $H_{m}\left(z, a^{1}, a^{2}\right)$ when $n \neq m$, we conclude that two-parameter analytic subgroups of $\Omega^{F}$ of different classes, although being locally isomorphic, are not globally isomorphic. 


\section{REFERENCES}

1. L. Bianchi, Lezioni sulla teoria dei gruppi continui finiti di trasformazioni, Spoerri, Pisa, 1918.

2. H. Cartan, Théorie élémentaire des fonctions analytique d'une ou plusieurs variables complexes, Hermann, Paris, 1961.

3. L. P. Eisenhart, Continuous groups of transformations, Dover, New York, 1961.

4. H. Hanani, A combinatorial identity, Amer. Math. Monthly. (to appear).

5. E. Jabotinsky, Sur la représentation de la composition de fonctions par un produit de matrices, C. R. Acad. Sci. Paris 224 (1947), 323-324.

6. - Analytic iteration, Trans. Amer. Math. Soc. 108 (1963), 457-477.

7. C. Jordan, Calculus of finite differences, 2nd ed., Chelsea, New York, 1960.

8. A. Ran, One parameter groups of formal power series, Duke Math. J. (to appear).

9. I. Schur, On Faber polynomials, Amer. J. Math. 67 (1945), 33-41.

ISRAEL INSTITUTE OF TECHNOLOGY, HAIFA, ISRAEL 